\title{
A visual tool for accessibility study of pipeline maintenance during design
}

\author{
Chu-Hsuan Lee ${ }^{1}$, Meng-Han Tsai ${ }^{2}$ and Shih-Chung Kang ${ }^{3 *}$
}

\begin{abstract}
Background: Pipeline maintenance is becoming an important issue in modern construction and building information model (BIM) research. An understanding of pipeline accessibility considerations in terms of operation and maintenance is essential for planning and management. Previous studies have highlighted the complexity of multi-pipes including mechanical, electrical and plumbing (MEP) pipelines and the importance of information visualization, but few have proposed a way to consider accessibility problems during operation and maintenance.
\end{abstract}

Methods: Therefore, this study develops a systematic method to evaluate accessibility with respect to pipeline maintenance. We first divided pipeline accessibility into three categories: (1) visual accessibility - the visibility for an inspector to view; (2) approachable accessibility - the difficulty for an inspector to approach; and (3) operational accessibility - the pipeline that can be operated by the inspectors. We created mathematical models and discussed the ergonomic details about each category. We then developed a user interface, VAO Checker, in which V, A and O stand for visual, approachable and operational respectively, to display visual information about pipeline accessibility. Through instantaneous analysis, the system visualizes the accessibility of the pipelines. We visually represent the intersection and union of these three categories to illustrate the varying accessibility of pipe elements.

Results: A usability test was conducted to validate the system's effectiveness. The results of the usability analysis show that users have higher correctness when using VAO Checker than 2D plan drawing and 3D model, and they evaluate the performance of this tool better than 2D plan drawing.

Conclusion: Pipeline designers can benefit by using this tool to sketch a suitable traffic flow for engineers to investigate. Furthermore, the substantial amount of information saved in the layout database could be referenced for future optimization.

Keywords: Pipeline maintenance; Building information model (BIM); Pipeline accessibility; Mechanical; Electrical; and Plumbing (MEP); Information Visualization

\section{Background}

Pipeline design has become increasingly important in modern construction. Operation and maintenance requires consideration of accessibility in the design of the layout of plant pipelines. Previous research has noted that piping accounts for $20 \%$ of costs for the industry as a whole (Calixto et al. 2009) and over 50\% of the total detail-design labor hours (Park and Storch 2002). All other activities of following detail design depend on piping and massive savings are achievable by utilizing good layout design and engineering practices.

\footnotetext{
* Correspondence: sckang@ntu.edu.tw

${ }^{3}$ Department of Civil Engineering, National Taiwan University, Taipei, Taiwan Full list of author information is available at the end of the article
}

Mechanical, electrical, and plumbing (MEP) pipes used to be supplemental facilities in construction. However, they have become necessary facilities, especially in nonresidential construction, such as hospitals, fire stations, and plants. Coordinating a MEP system is a tremendous challenge in engineering fields such as advanced technology, health care, and biochemistry industries (Khanzode et al. 2008). Knowing how to arrange MEP systems appropriately is one of the most crucial aspects of the design phase (Riley et al. 2005).

Maintenance is a crucial phase in these types of construction. Based on a statistics on expenses in a typical water treatment plant in year-2008, maintenance takes 15\% possession of the expenses (Biehl and Inman 2010). 
Moreover, based on some European and U.S. case study, maintenance has remarkable impact on at least one of the environmental aspects (Junnila et al. 2006). A poorly designed pipeline layout design wastes space and materials. Moreover, it can cause difficulty or even danger during manipulation and management.

\section{Literature review}

The literature reviewed for this study included findings and recommendations related to piping that can be categorized into three main groups: a pipe-routing algorithm, the integration of multi-pipes, and the visualization of pipeline design.

\section{Pipe-routing algorithms}

Pipe-routing design is a subset of assembly design that conceives collision-free routes for pipes. A survey by Qian et al. (2008) categorized it into four fields: industrial plant pipeline layout design, circuit layout design, aircraft design, and ship piping system design (Qian et al. 2008). Several studies have been devoted to routing algorithms, and mainly focus on physical constraints that connect the terminals of given locations and avoid all obstacles. They then use economic constraints to minimize the length of pipes and the number of pipe turns, which leads to an optimal specification. However, few, if any, solutions have considered pipeline accessibility in relation to operation and maintenance. Table 1 shows previous studies have disregarded some important constraints (Guirardello and Swaney 2005; Ito 1999; Mitsuta et al. 1987; Newell 1972; Park and Storch 2002; Rourke 1975; Schmidt-Traub et al. 1998; Wangdahl et al. 1974; Zhu and Latombe 1991). Zhou and Yin (2010) emphasized that practical constraints, such as maintenance requirements and manufacturability, are not well recognized, and how humans still play an important role in guiding the computer to finish the design (Zhou and Yin 2010).

\section{Integration of multi-pipes}

An industrial plant typically has more than one kind of pipeline. Feng et al. (2012) indicated a large number of pipelines, multifarious design constraints, and numerous obstacles in layout complicate the design of a pipeline system (Feng et al. 2012). Recently, engineers have mainly used existing CAD software for design assistance, which has increased the problems associated with experts, such as complex operation, a long design cycle, and low efficiency. Some researchers advocated a new layout space model to reduce high complexity and design interference in the automated design of pipeline systems (Deliang and Huibiao 2009; Feng et al. 2012). Kim et al. (1996) found the range and complexity of the constraints limits the possibility of automatic pipe route design, and demonstrated a more natural and effective representation for route optimization (Kim et al. 1996). Previous studies recognized the complexity in pipeline arrangement and proposed some methods to reduce it (Biehl and Inman 2010; Guirardello and Swaney 2005). However, in many instances the pipeline layout cannot be simplified, so the complexity should be taken into account.

\section{Visualization regarding pipeline accessibility}

Some researchers have begun noticing the utility of information visualization for construction purposes as a means of improving the data-rich, but information-poor, problems of the construction industry (Songer et al. 2004; Tsai et al. 2010; Tsai et al. 2013). Previous research focused on the visualization of construction data, noting how it can help identify potential causal relationships among construction data (Korde et al. 2005; Kuo et al. 2011; Russell et al. 2009). Gao et al. (2006) investigated colored construction drawing, which can increase the efficiency and accuracy of communication between designers and contractors (Grootjans 2009a). Chang et al. (2009) and Chen et al. (2013) suggested a systematic procedure to determine the most suitable colors for effectively presenting the construction information (Chang et al. 2009; Chen et al. 2013). This procedure includes the selection, evaluation, and testing of colors to ensure they match the meaning of the construction information with the cognition of the users. Wang (2011) used the conception of visualization to develop an approach for

Table 1 Earlier studies of pipe routing

\begin{tabular}{|c|c|c|c|c|c|c|c|c|}
\hline \multirow{2}{*}{$\frac{\text { Algorithm }}{\text { Author }}$} & \multicolumn{4}{|c|}{ Network optimization } & \multicolumn{2}{|l|}{ Maze } & \multirow{2}{*}{$\begin{array}{l}\text { Escape } \\
\text { Schmidt }\end{array}$} & \multirow{2}{*}{$\begin{array}{l}\text { Genetic } \\
\text { Ito }\end{array}$} \\
\hline & Newell & Wangdahl & Zhu & Guiradello & Rourke & Mitsuta & & \\
\hline Year & 1972 & 1974 & 1991 & 2005 & 1975 & 1987 & 1998 & 1999 \\
\hline Dimension & $3 \mathrm{D}$ & $2 \mathrm{D}$ & 2D/3D & $3 \mathrm{D}$ & $3 \mathrm{D}$ & $3 \mathrm{D}$ & $3 \mathrm{D}$ & $2 \mathrm{D}$ \\
\hline Domain & General & Ship & Robotics & Plant & General & General & Plant & General \\
\hline Operation/maintenance & - & - & - & - & - & - & - & - \\
\hline Installation & - & - & ० & - & - & - & ० & ० \\
\hline Safety & - & - & - & - & - & $\bullet$ & - & - \\
\hline
\end{tabular}

-: not considered, ○: partially implemented, $\bullet$ : fully implemented. 
assessing reachability of wheelchair users (Wang 2011). With reference to pipeline arrangement, Deliang and Huibiao (2009) pointed out that visualization can help handle the detection and response to collisions between pipes and obstacles (Deliang and Huibiao 2009).

\section{Expert interviews}

During the early stage of this research, we interviewed six experts to determine the requirements of pipeline design. They are all in the field of plant pipeline design, including three engineers from a construction company, two managers from a microelectronics corporation, and one executive officer from the Building Information Modeling (BIM) research center. After combining the opinion of experts with previous literature review, we mainly focused our research on pipeline accessibility during operation and maintenance, which is rarely discussed in previous studies.

\section{Needs analysis}

We determined from the interviews that there are four main considerations in pipeline design: (1) the manufacturing process, (2) operation and maintenance, (3) cost, and (4) aesthetics. In a typical plant engine room, as depicted in Figure 1, the engineers first have to deliberate how the pipelines go according to the manufacturing process, which will influence productivity and efficiency. They then contemplate how the workers will handle the equipment, meters, and valves during the operation and maintenance phase. Cost and aesthetics are aspects used to optimize the consequences of designs. Previous studies have proposed many algorithms by considering the cost factor, but maintenance is rarely discussed.

We mainly focused on operation and maintenance. Pipeline accessibility is the key factor to effective maintenance as it determines how easily the engineers can stretch to the accessories related to pipelines, including equipment, meters, and valves. Engineers can sometimes

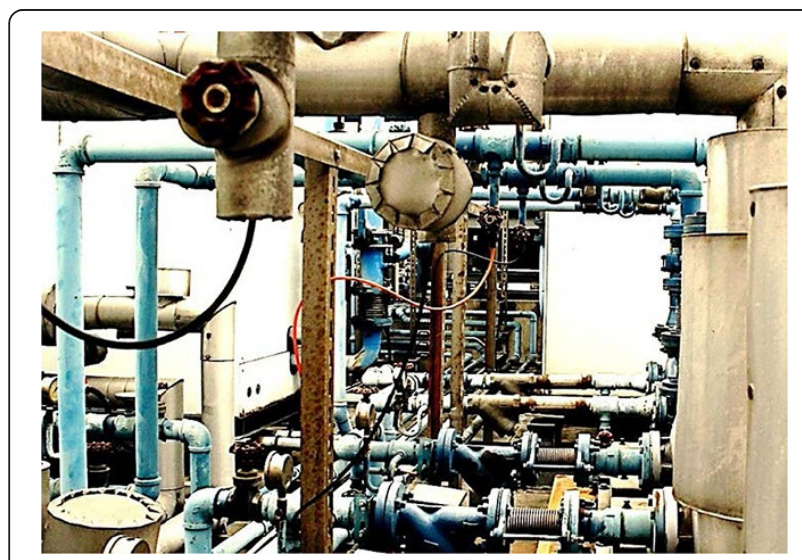

Figure $1 \mathrm{~A}$ typical pipeline arrangement in an engine room. see pipelines from a distance, but cannot approach them due to the obstacles in the way of the pipelines. In other cases, engineers cannot read the meters in detail or operate the valves without difficulty, because these parts are mounted too high. We seek an easy way to illustrate pipeline accessibility with a view to engineers benefiting from this intuitive tool during the construction cycle (i.e., design, operation, and maintenance).

\section{Objective and scope}

The aim of this study is to develop a method to assist decisions about pipeline maintenance. One major challenge of coordinating MEP multi-pipes is identifying the spatial conflicts between systems. Through instantaneous analysis, the system automatically produces visual information indicating how much pipe access the engineers can have. This tool allows users to view, explore, and interact with the pipeline information via a direct manipulation interface in order to identify the spatial accessibility in a more intuitive manner. The user can thus obtain a comprehensive understanding of pipeline maintenance.

\section{Methods}

We use a Venn diagram ${ }^{\mathrm{a}}$, a diagram that shows all possible logical relations between different sets, to differentiate three categories of pipeline accessibility. We then apply each section of the diagram to different scenarios. We further develop mathematical models and discuss the ergonomic details about each different category.

\section{Overall procedure of pipeline accessibility}

We proposed three categories, visual, approachable, and operational to present the extent to which the pipe

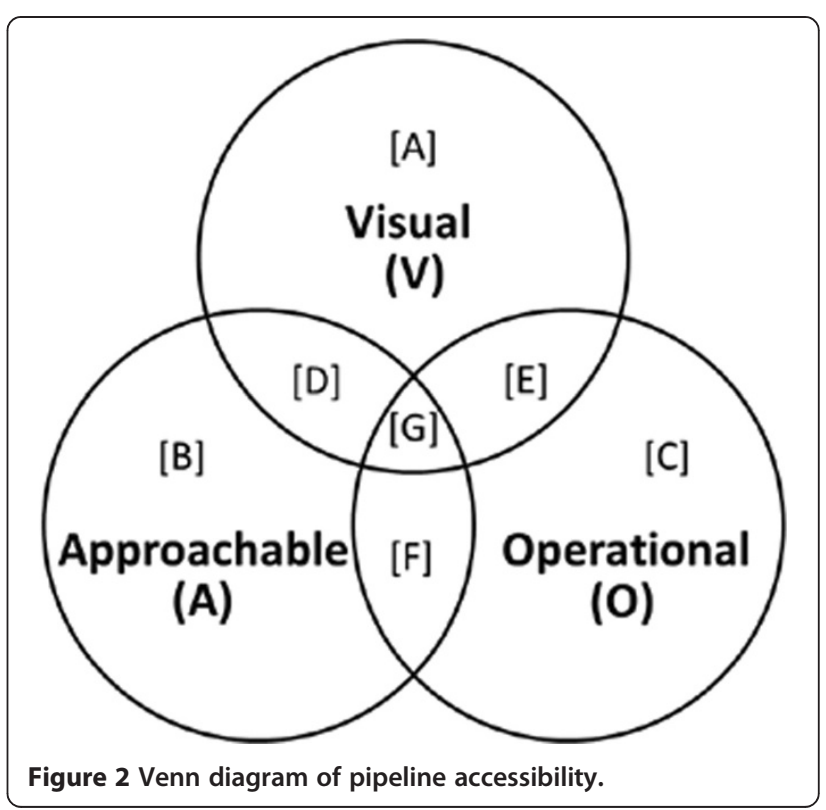


Table 2 Seven variations of intersection and union

\begin{tabular}{|c|c|c|}
\hline Section & $\begin{array}{l}\text { Math } \\
\text { representation }\end{array}$ & Accessibility description \\
\hline$[\mathrm{A}]$ & $V-A-O$ & $\begin{array}{l}\text { Only visible, but not approachable and } \\
\text { operable. This happens when obstacles and } \\
\text { other pipes prevent engineers from } \\
\text { accessing equipment and pipelines. }\end{array}$ \\
\hline$[B]$ & $A-V-O$ & $\begin{array}{l}\text { Only approachable, but not visible and } \\
\text { operable. This happens when obstacles and } \\
\text { other pipes block displays and controls. }\end{array}$ \\
\hline$[C]$ & $O-V-A$ & $\begin{array}{l}\text { Only operable, but not visible and } \\
\text { approachable. Although remote control is } \\
\text { possible, we did not consider this variation. }\end{array}$ \\
\hline [D] & $V \cap A-O$ & $\begin{array}{l}\text { Visible and approachable, but not operable. } \\
\text { This happens when controls or valves are } \\
\text { mounted too high, too low, or too far away } \\
\text { to reach and operate. }\end{array}$ \\
\hline$[\mathrm{E}]$ & $\mathrm{V} \cap \mathrm{O}-\mathrm{A}$ & $\begin{array}{l}\text { Operable and visible, but not approachable. } \\
\text { The same as [C]. We did not consider this } \\
\text { variation. }\end{array}$ \\
\hline$[\mathrm{F}]$ & $\mathrm{V} \cap \mathrm{O}-\mathrm{V}$ & $\begin{array}{l}\text { Approachable and operable, but not visible. } \\
\text { This happens when controls and valves are } \\
\text { mounted behind the display, and engineers } \\
\text { have to bend their arms to operate them. } \\
\text { However, any blindness operation is not } \\
\text { allowed in our assumption. }\end{array}$ \\
\hline [G] & $V \cap A \cap O$ & $\begin{array}{l}\text { Visible, approachable, and operable-the } \\
\text { ideal situation. }\end{array}$ \\
\hline
\end{tabular}

elements are accessible. As shown in Figure 2, we use the intersection and union of these three categories to discuss different scenarios as follows:

Visual (V): determines how much of the pipe is directly visible for inspection.

Approachable (A): determines how far maintenance engineers can walk along the pipes.

Operational $(\mathrm{O})$ : checks how much of the pipes can be reached in order to operate valves or check surfaces.

In the Venn diagram, there are seven sections among the three circles. Each one is a variation of intersection and union. As listed in Table 2, we give the accessibility description of each variation from Figure 2 .

These three categories are expressed in a visual conception of information. We adopted the anthropometric data from the American Bureau of Shipping (The American Bureau of Shipping 2003) to build the model for accessibility analysis. We made some modifications by considering the physical differences between Americans and Taiwanese, because the first case would be a semiconductor fabrication plant in Taiwan.

\section{Approachable accessibility}

This level determines how far people can walk along the pipes. Walkways should have $2.1 \mathrm{~m}$ minimum clearance above the walking surface for the full length and width of the walkway. The analysis and mathematical model of approachable accessibility is different from the other two because it is a dynamic process. As shown in Figure 3 and Table 3, we first use a bounding cylinder to represent a

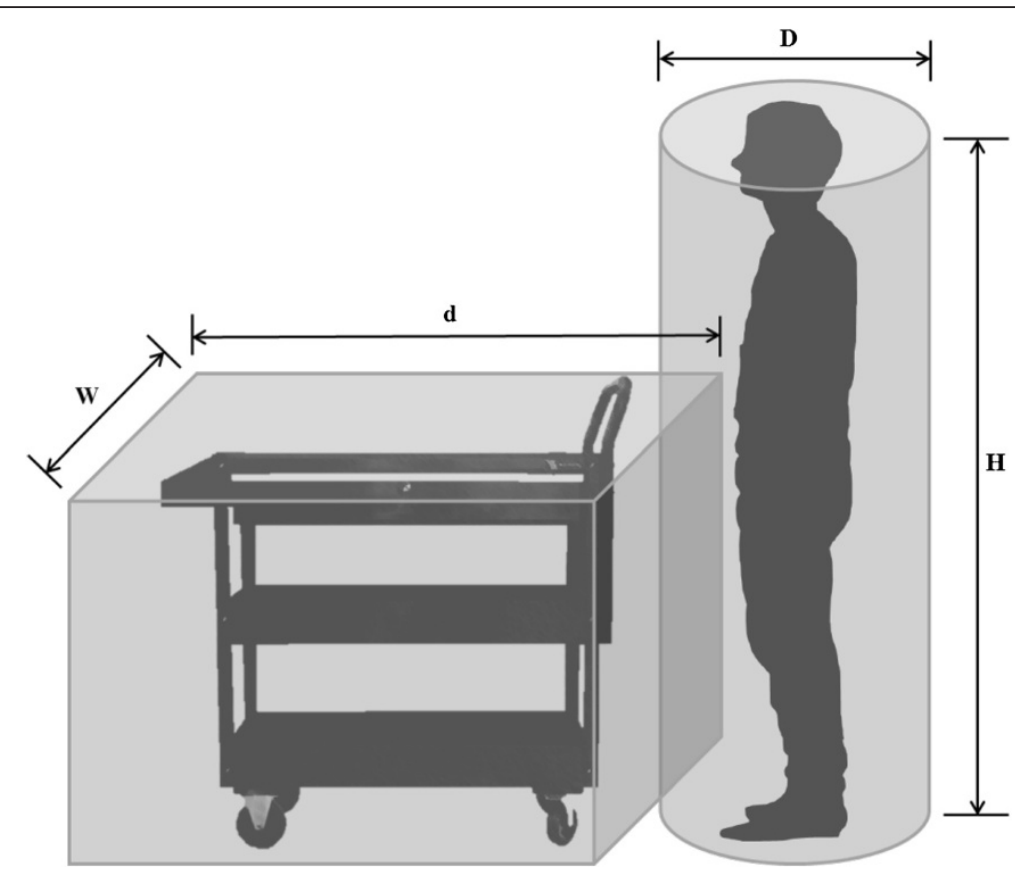

Figure 3 Bounding cylinder and box representation. 
Table 3 Bounding box size for recommended walkway dimensions

\begin{tabular}{ll}
\hline Application & Box size $^{*}$ \\
\hline One person traveling in an area with limited access & $51 \times 51 \times 150$ \\
$\begin{array}{l}\text { One person in unrestricted area, where two persons } \\
\text { could pass }\end{array}$ & $71 \times 71 \times 210$ \\
One person with a cart & $71 \times 120 \times 210$ \\
$\begin{array}{l}\text { Normal two-way traffic or any means of egress that } \\
\text { leads to an entrance or exit }\end{array}$ & $92 \times 120 \times 210$ \\
Corridor or passageway that serves as a required exit & $112 \times 120 \times 210$ \\
\hline
\end{tabular}

*Size representation: $\mathrm{W}(\mathrm{cm}) \times(\mathrm{D}+\mathrm{d})(\mathrm{cm}) \times \mathrm{H}(\mathrm{cm})$.

person, and bounding boxes in different sizes to represent a cart in different applications. If obstacles or other pipes block the box, it cannot go farther along the pipes.

The mathematical model of visual accessibility is then constructed as the equation:

$$
A=(H, r, P)
$$

As denoted in Figure 4, $r=\frac{\operatorname{Max}(W, D)}{2}$, and we used a cylinder with radius $r$ and height $H$ to simplify the bounding box. $S$ means the start point, and $T$ means the target point. $P$ is the path from $S$ to $T$ :

$P=\left[S, p_{1}, p_{2}, \ldots, p_{n}, p_{n+1}, \ldots T\right]$, where the cylinder is not blocked.

\section{Visual accessibility}

This level determines how much of the pipe is directly visible for inspection. We further divide it into two levels: visible and legible. The former includes those used for normal operations and those not requiring accurate readings, whereas the latter includes those used frequently, for obtaining precise readings, and in emergencies. The mathematical model of visual accessibility is constructed as the following equation. Figure 5 indicates the parameters.

$$
V=\left(S,{ }^{o} H,{ }^{o} L_{\text {min }},{ }^{o} L_{\text {max }}, \theta,{ }^{o} H_{\text {min }}^{v}, H_{\text {max }}^{v}\right)
$$

Figure 6 indicates people's field-of-view. The two parameters regarding it are the distance from eyes $(L)$ and the viewing angle from the central line $(\theta)$. Based on ABS research, as shown in Table 4, people can see the details of pipes at distances between $33 \mathrm{~cm}$ and $71 \mathrm{~cm}$, and a viewing angle within 35 degrees, where the legible level should be located (provided obstacles or other pipes do not block the pipes and displays). The distance for the visible level can be up to $200 \mathrm{~cm}$, with the viewing angle up to 60 degrees. Figure 7 illustrates the visual heights $\left(H^{\nu}\right)$ for displays in different postures: standing $(\mathrm{C})$, kneeling (D), and squatting (E). Table 5 shows the maximum and minimum heights for the legible and visible levels, based on personal height $(\mathrm{H})$. Because the range of these three postures overlapped, we integrated the data. The legible level should be located within the multiple 0.4261 0.9375 , but the visible level can be broader, 0.2955-1.0114.

\section{Operational accessibility}

To facilitate the operation of valves or the checking of surfaces, this level checks the accessibility of pipes. It is derived from the arrival accessibility level, and shows the ease with which people can operate within the pipe layout. We further divided it into two levels: general control and precise control. The former includes those used for normal operations and those not requiring accurate manipulation, whereas the latter includes those used frequently, for obtaining precise performance, or in emergencies. The mathematical model of operational accessibility is constructed as the following equation. Figure 8 indicates the parameters.

$$
O=\left(S,{ }^{o} H,{ }^{o} F,{ }^{o} H_{\min }^{o}, H_{\max }^{o}\right)
$$

People's forward functional reach from behind the shoulder to the tip of the extended finger $(F)$ and the operable heights $\left(H^{0}\right)$ for controls in different postures are illustrated in Figure 7: standing (G), kneeling (I), and squatting (J). Table 6 shows the maximum and minimum forward functional reach and heights for precise and general controls, based on personal height $(H)$. Frequently used controls should be located within a radius of multiple 0.2614 from the operator's centerline, whereas less frequently used controls should be located within a radius of multiple 0.4545

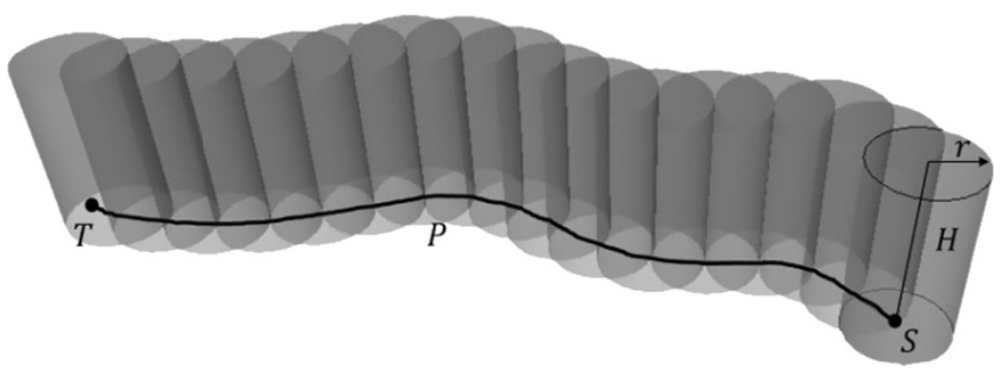

Figure 4 Mathematical model of approachable accessibility. 


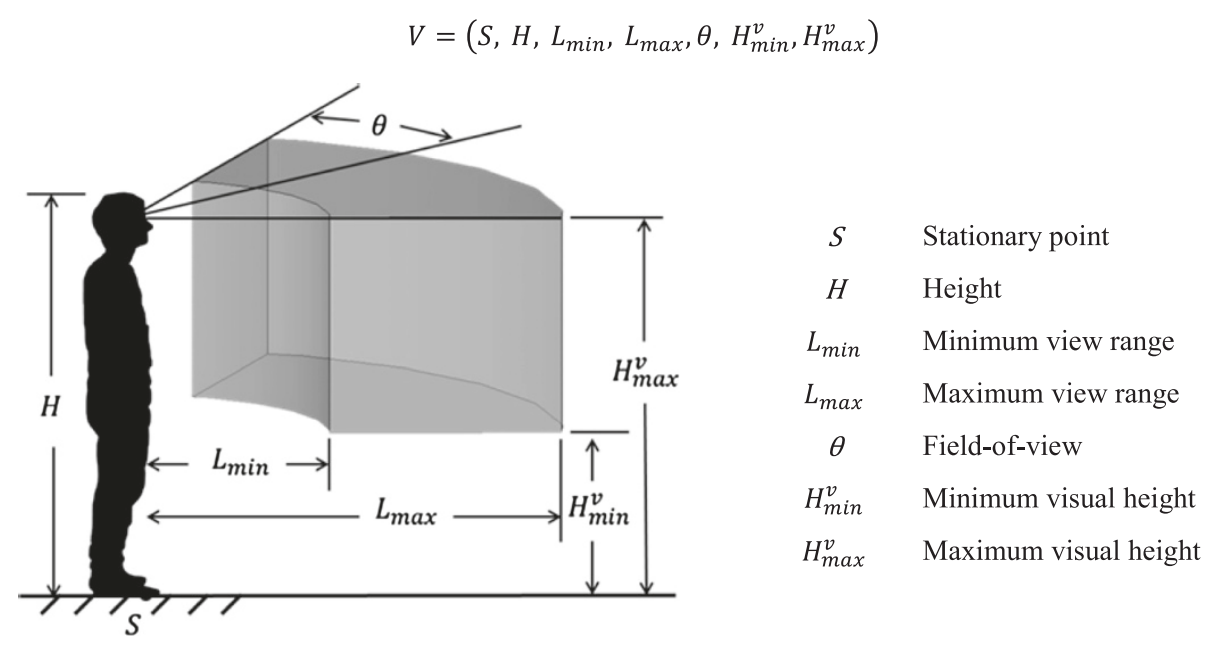

Figure 5 Mathematical model of visual accessibility.

from the operator's centerline. Because the range of these three postures overlapped, we integrated the data. Precise control should be located within the multiple 0.22730.7670, but general control can be broader, 0.2045-1.0966.

\section{Implementation}

This study developed a system, VAO Checker, which integrated the user interface and visualization information as a tool, to implement the proposed methodology. The

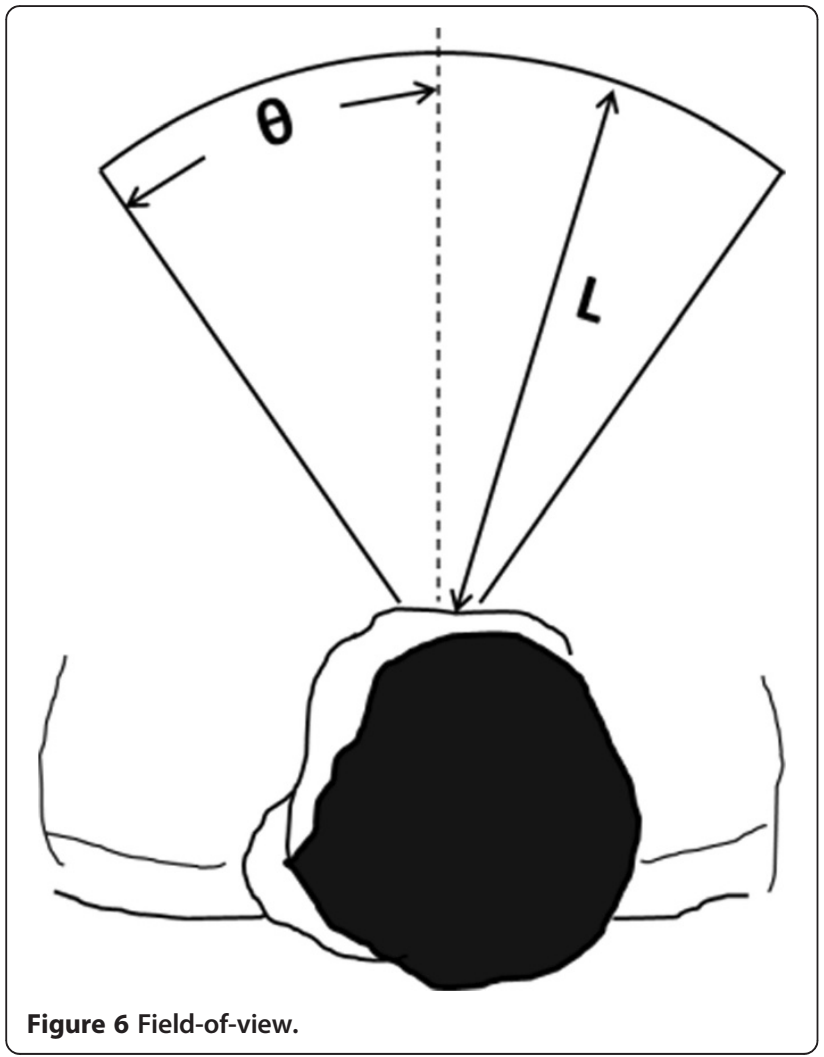

following sections describe the software used for the development environment and the system design.

\section{Programming platform}

This study used Microsoft Windows Presentation Foundation (WPF) for the display of the user interface. WPF was chosen because it allows programmers to easily unify multimedia data, and change the appearance or the function of display controls for customization. Furthermore, the WPF application functions by off-loading to graphics processing units (GPUs) rather than central processing units (CPUs), which facilitates smoother graphics and better performance (Nathan 2006).

\section{Graphics engine}

The framework developed for the visualization information was based on the Microsoft XNA Game Studio 4.0. This tool assists the development of video games and the improvement of software management. XNA has ample performance for the development of $2 \mathrm{D}$ and $3 \mathrm{D}$ games. It offers users the capability to build the operating system and visual images with ease (Grootjans 2009; Miller and Johnson 2010).

\section{System design}

The proposed tool called VAO Checker was built for this study to consider the three categories of pipeline accessibility. As shown in Figure 9, the operation interface

Table 4 Suitable range of field-of-view

\begin{tabular}{lll}
\hline & $\mathbf{L}(\mathbf{c m})$ & $\boldsymbol{\theta}$ (degrees) \\
\hline Legible & $33-71$ & 35 \\
Visible & $0-200$ & 60 \\
\hline
\end{tabular}




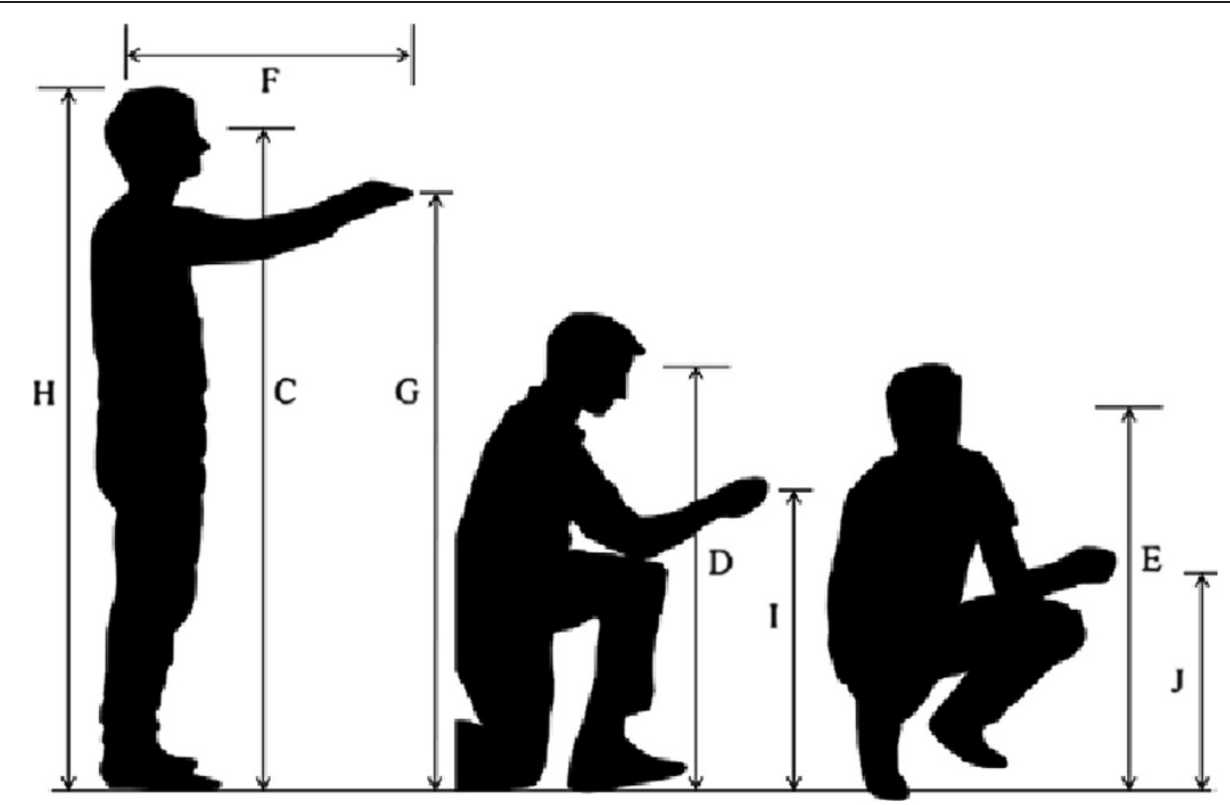

Figure 7 Related height in different postures.

displays a plan view of the space, including the equipment and pipelines. The user can use this tool to find a collision-free path through the space and to examine the different levels of visual and operational accessibility.

\section{Example case}

We created an example case to validate the practicality of VAO Checker. We built a virtual building project as an example case in a machinery room (Figure 10), which has some basic equipment and a pipeline arrangement.

After the start point and target point are decided, and the "Approachable" button is clicked, a collision-free path with some check points listed in the blank comes into view (Figure 11). The dot with sufficient approachable accessibility is bigger and colored light green, and the dot with limited approachable accessibility is smaller and colored dark green.

The user can choose one of those listed points, and the chosen point will turn into a red dot (Figure 12). The user can then examine different levels of visual and operational accessibility by clicking the four buttons at the bottom right corner. A visualization window, indicating a corresponding level of accessibility, will open (Figure 13). The user can utilize some specific keys to interact with the pipeline information, such as rotating the view direction or stepping forward or backward.

\section{Validation}

In order to verify how VAO Checker could help users explore and understand relevant accessibility information, we conducted a usability test. We also solicited expert consultation to verify the usability and how the users can interact with the pipeline accessibility information.

\section{Test plan}

Test procedure For the usability test, we built a typical machinery room project with equipment and pipelines. There were 10 accessibility problems in this case, which are categorized in Table 7 according to Figure 2 and

Table 5 Suitable field-of-view and vision range (multiple of $\mathrm{H}$ ) for legible and visible levels

\begin{tabular}{lllllll}
\hline Posture & $\mathbf{L}(\mathbf{c m})$ & $\boldsymbol{\theta}$ (degrees) & Standing (C) & Kneeling (D) & Squatting (E) & Overall \\
\hline Visible maximum & 200 & 60 & 1.0114 & 0.8239 & 0.7102 & 1.0114 \\
Legible maximum & 71 & 35 & 0.9375 & 0.7500 & 0.5795 & 0.9375 \\
Legible minimum & 33 & 0 & 0.7216 & 0.5398 & 0.4261 & 0.4261 \\
Visible minimum & 0 & 0 & 0.5909 & 0.3977 & 0.2955 & 0.2955 \\
\hline
\end{tabular}




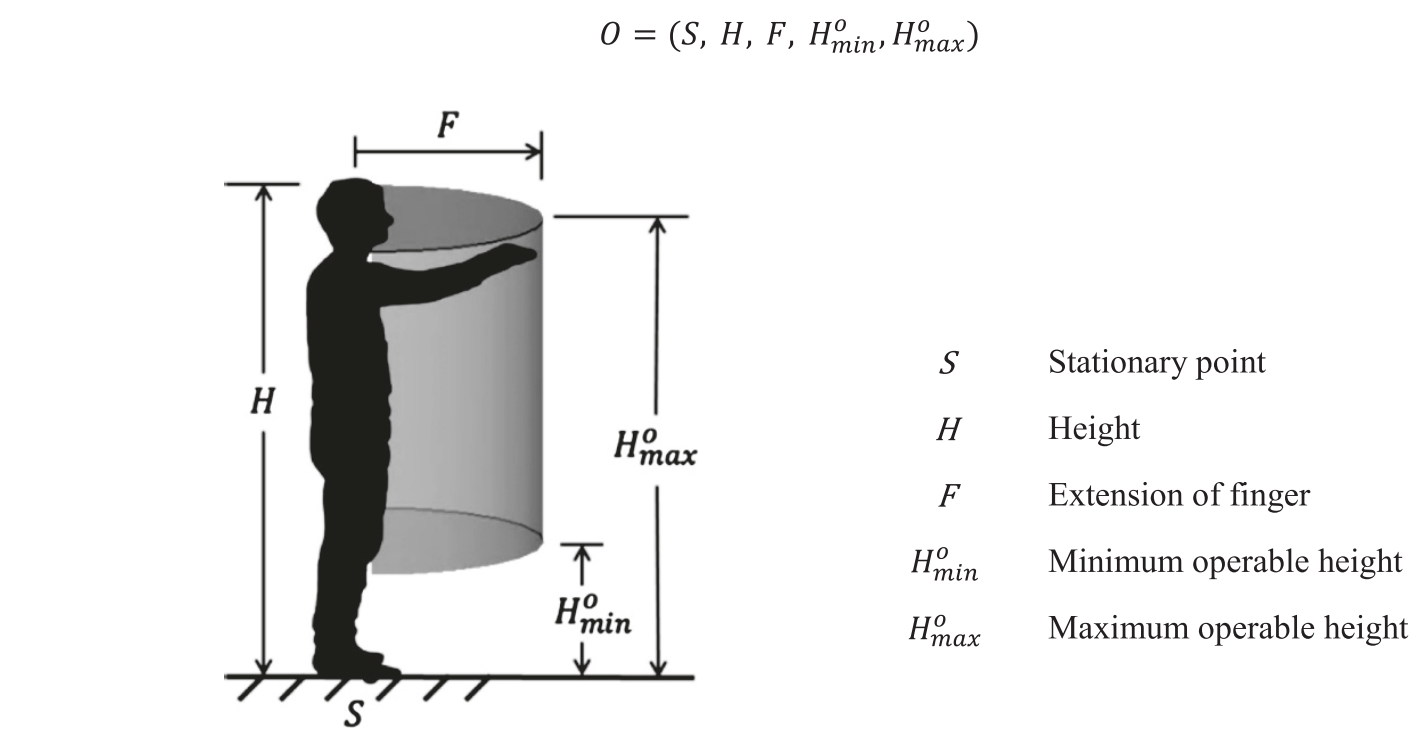

Figure 8 Mathematical model of operational accessibility.

Table 2. All users had to identify the problems in three individual tasks, each task using different mediums, 2D plan drawing, 3D model and our system, VAO Checker. Besides, we also conducted the NASA Task Load Index (NASA-TLX) test. As shown in Figure 14, the test plan began with the NASA-TLX weight assessment, in which the user compared the factors pairwise based on their perceived importance. After the user finished the identification of accessibility problems via one information medium, the user had to rate each factor of task load within a 100-points range. The final NASA-TLX score was calculated based on the weight distribution, which was decided at the initial phase.

Test participants There were 30 participants in the usability test, 19 male and 11 female. Their ages ranged from 20 to 37 years old. We solicited participant from nonengineering background as well, because they might provide suggestion from different point of view. Among the participants, 27 participants are from a civil engineering background, 2 from a psychology background, and 1 from an economics background.
Test environment The usability test was conducted in a controlled environment that was limited to the room shown in Figure 15. In this room, each participant was asked to sit at the east side of the front table in the room. A researcher, sitting next to the participant, conducted and facilitated the test procedure and guided the participants through the test.

\section{Results and discussion}

An $\alpha$ level of 0.05 was used for all statistical tests and analysis, and we calculated the $\mathrm{p}$-value between groups in analysis of variance (ANOVA), where $p<0.05$ means statistically significant. The test results assessed how quickly and accurately participants performed the task when using different mediums. There is also an analysis of NASATLX score, which shows how the participants evaluated the ergonomics performance of each medium. They are summarized as follows:

Correctness: $V A O \geqq 3 D>2 D$

The box-and-whisker plot, a visual display of the five number summary, of success rate of each medium is shown in Figure 16. Table 8 presents means and

Table 6 Suitable forward functional reach and heights (multiple of H) for precise and general controls

\begin{tabular}{llllll}
\hline Posture & Forward (F) & Standing (G) & Kneeling (I) & Squatting (J) & Overall \\
\hline General maximum & 0.4545 & 1.0966 & 0.8239 & 0.7102 & 1.0966 \\
Precise maximum & 0.2614 & 0.7670 & 0.6136 & 0.4545 & 0.7670 \\
Precise minimum & 0 & 0.4886 & 0.3068 & 0.2273 & 0.2273 \\
General minimum & 0 & 0.4318 & 0.2614 & 0.2045 & 0.2045 \\
\hline
\end{tabular}




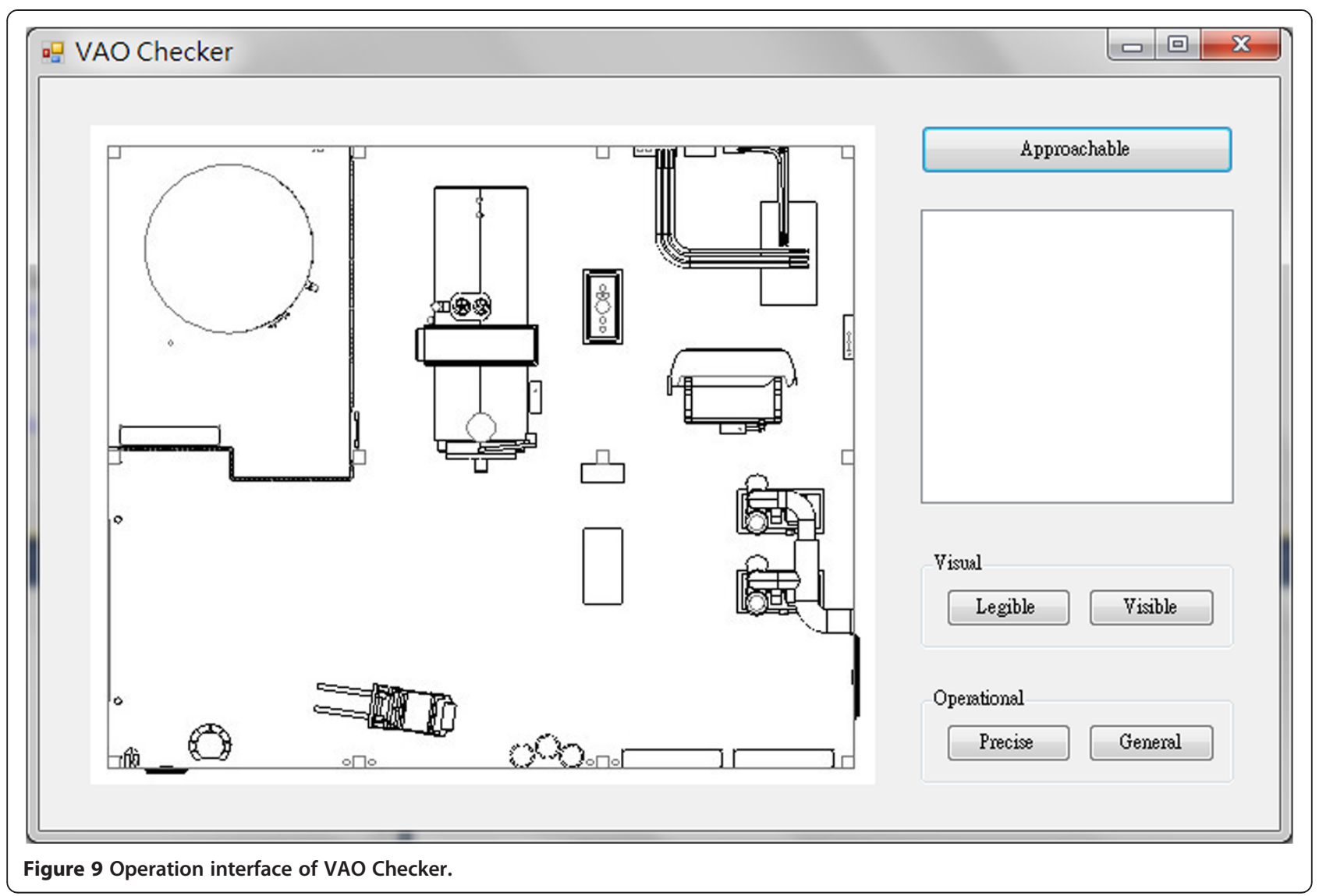

standard deviations of success rate of each medium, and the p-value shows the data between 2D and VAO Checker is statistically significant. As the data indicates, the success rate of VAO Checker (64.3\%) is 1.6 times higher than 2D plan drawing (40.1\%) and 1.14 times higher than 3D model (56.4\%).
Performance: $3 D>V A O>2 D$

The box-and-whisker plot of NASA-TLX score of each medium is shown in Figure 17. Table 9 presents means and standard deviations of NASA-TLX score of each medium, and the $\mathrm{p}$-value shows the data between each pair of these three groups is statistically significant. The

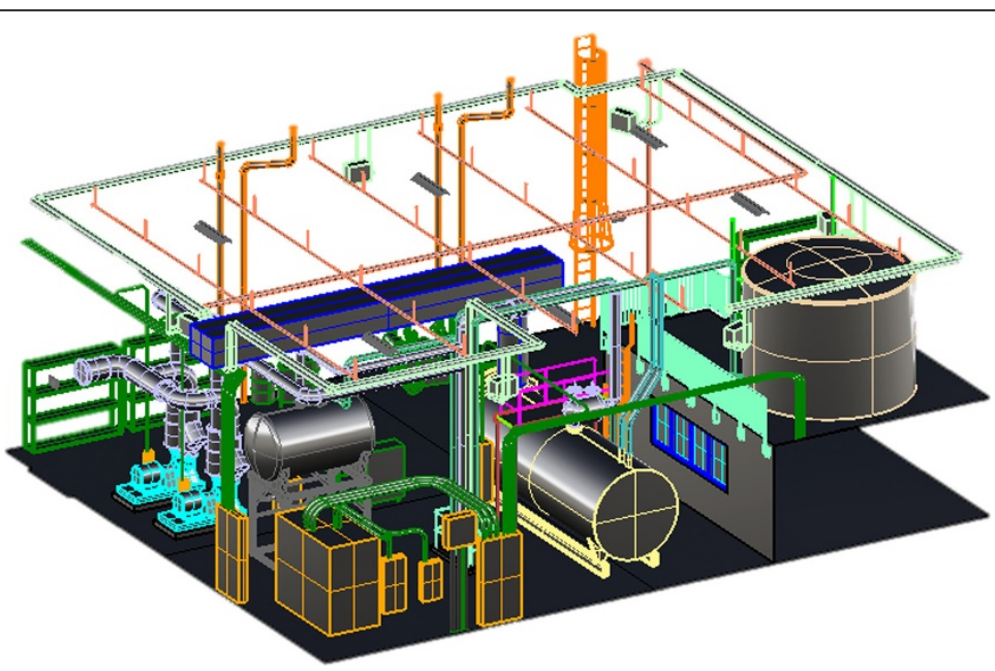

Figure 10 Example case. 


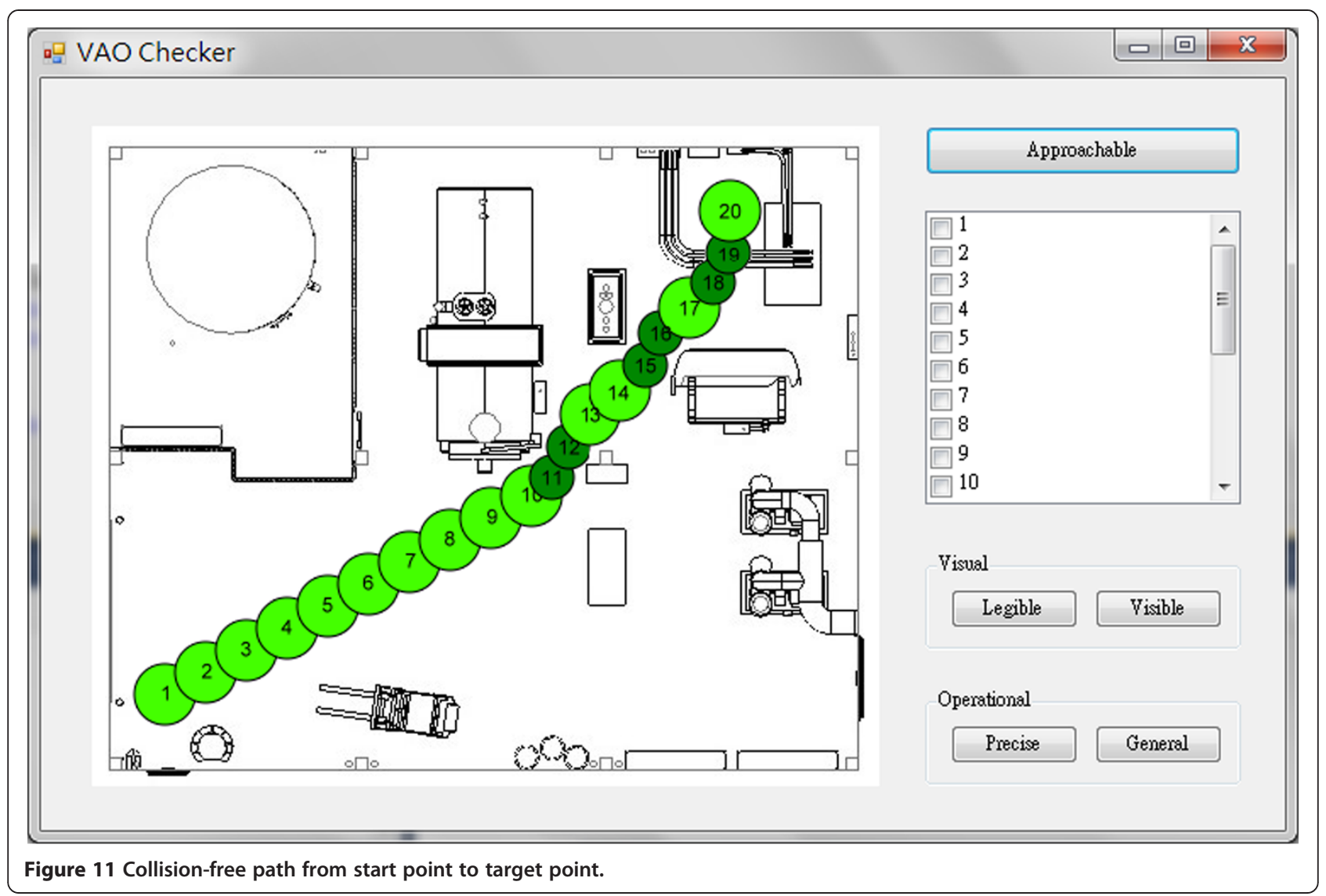

score of 2D plan drawing is the lowest (36.0), whereas the score of 3D model is the highest (53.8). The score of VAO Checker (48.0) is 1.33 times higher than 2D plan drawing.

\section{Findings from the result}

Most of the participants have a background of civil engineering, and they can get on track quickly when they check 2D plan drawing or 3D model. Based on the observation during the usability test, participants would spend some time to get used to the user interface of VAO Checker, because it is a new tool for them. As a result, the average problem identification time of VAO Checker is longer than $2 \mathrm{D}$ plan drawing and 3D model.

However, in the analysis of correctness, the success rate of VAO Checker is the highest. This means, although users might spend more time when they first contact with the user interface of VAO Checker, they still can achieve the goal of high correctness. Some participants advised that in addition to the three categories of accessibility, VAO Checker should take more safety factors into consideration. They indicated that the section where steam is generated should be prohibited to pass through. Then, the path should bypass those areas.

In the analysis of performance, the NASA-TLX score of $3 \mathrm{D}$ model is slightly higher than VAO Checker. We also interviewed the participants about their feeling when they manipulated VAO Checker. Many of them pointed out that the manipulation of VAO Checker had a sense of reality, unlike $2 \mathrm{D}$ plan drawing. They could look around the environment, and perceive the size of equipment and pipelines. The visual effects made it like playing a game. However, because the viewing angle of VAO Checker is set as first person, they sometimes got confused with the direction in the virtual environment. On the contrary, the viewing angle of 3D model is set as third person, and they can identify the direction easily. That is the reason some participants evaluated the NASA-TLX score of 3D model higher.

Furthermore, many of the participants commented that another reason they got confused with the direction is the unfamiliarity with the overall pipeline design. VAO Checker would serve as a useful tool for the designers who are conscious of the design, and they would benefit from this tool to correct any design 


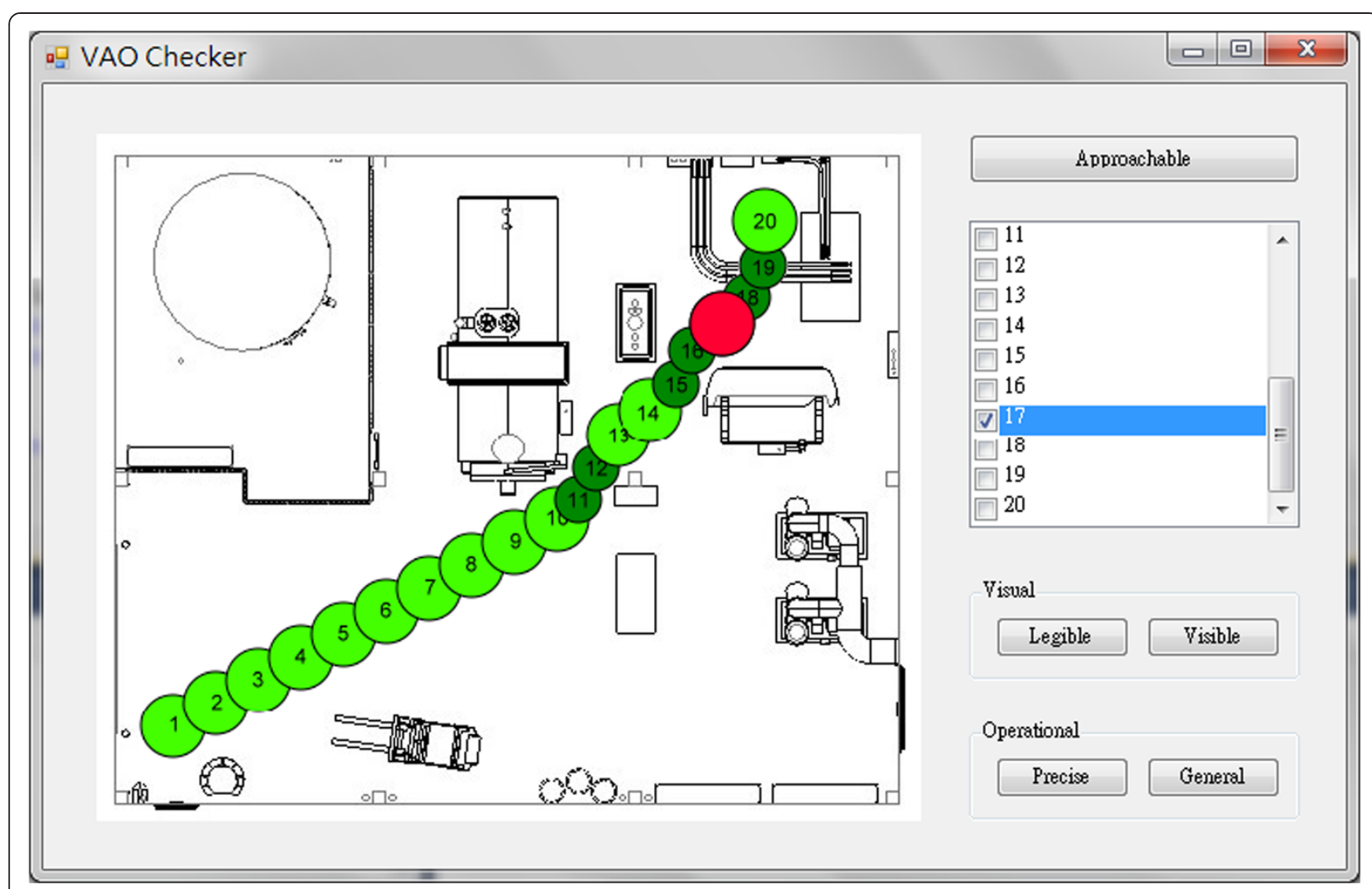

Figure 12 Chosen check point turns into a red dot.

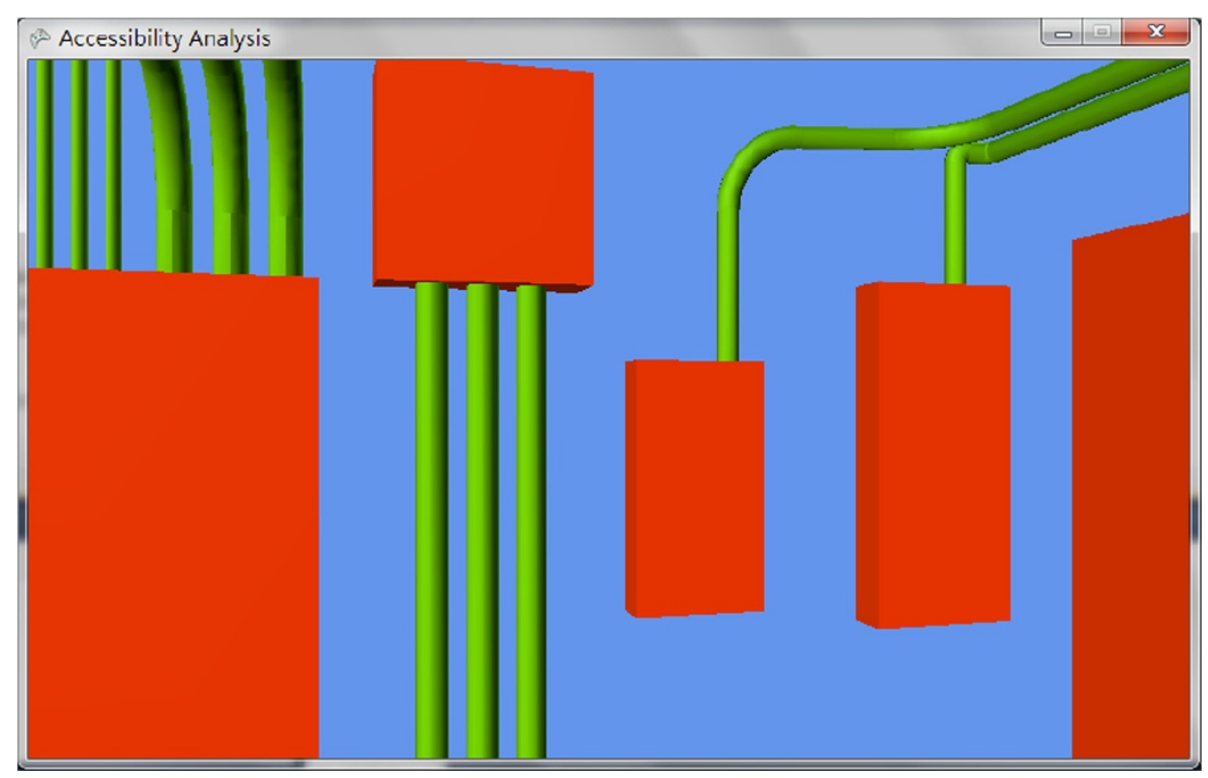

Figure 13 Visualization window indicating corresponding level of accessibility. 
Table 7 Category of 10 accessibility problems

\begin{tabular}{lllllllll}
\hline Category & {$[\mathrm{A}]$} & {$[\mathrm{B}]$} & {$[\mathrm{C}]$} & {$[\mathrm{D}]$} & {$[\mathrm{E}]$} & {$[\mathrm{F}]$} & {$[\mathrm{G}]$} & Total \\
\hline Amount & 3 & 2 & - & 3 & - & 2 & - & 10 \\
\hline
\end{tabular}

errors. They suggested that VAO Checker is suitable for planning a more complex environment, such as chiller machinery room. The sizes of pipelines are bigger, and there are more relevant systems. Formerly only experienced designers could plan a pipeline layout which is acceptable enough. Through VAO Checker, designers could save a lot of time in analyzing and planning.

Owing to the booming BIM industry, some participants supposed VAO Checker would be developed into an application-programming interface (API) of BIM related software. Construction companies or corporations are increasingly using BIM as a tool to integrate information from the fields of architecture, structural engineering, and MEP systems. If VAO Checker can be developed into an API, more pipeline designers and engineers can benefit by saving time and effort.

Despite the participants needed some time to be familiar with the manipulation interface of VAO Checker, they all agreed that they could identify the accessibility problems very easily via this tool, because it provided sufficient information for them to judge the level of pipeline accessibility. They expected the path generated from analysis of approachable accessibility could be used for inspection or judgment, and the engineers would have a certain understanding of pipeline maintenance of the entire environment if they could move along this path.

\section{Contribution}

Unlike previous studies, in which few solutions have been offered to propose a way to consider pipeline accessibility through maintenance, our research emphasizes the importance of pipeline accessibility and makes efforts on the following sections:

\section{A useful tool for pipeline designers and engineers}

We have shown that VAO Checker serves as a useful system for pipeline designers and engineers during operation and maintenance. Designers can benefit by sketching a suitable traffic flow for the users' investigation. Engineers can obtain a comprehensive understanding of the pipeline maintenance with the aid of the interface and can identify spatial accessibility in a more intuitive manner.

\section{Considering pipeline accessibility}

Unlike previous studies, in which few, if any, solutions have been offered to propose a way to consider pipeline accessibility through operation and maintenance, our research emphasizes its importance.

\section{Integration of information from multi-pipes}

With regard to the complexity of multi-pipes, different kinds of pipes might have to be considered in terms of different levels of accessibility. We developed mathematical models about each different accessibility category and discussed the ergonomic details.

\section{Enhancing comprehension via visualization}

Given the importance of visualization for pipeline accessibility, we developed VAO Checker, which integrated the

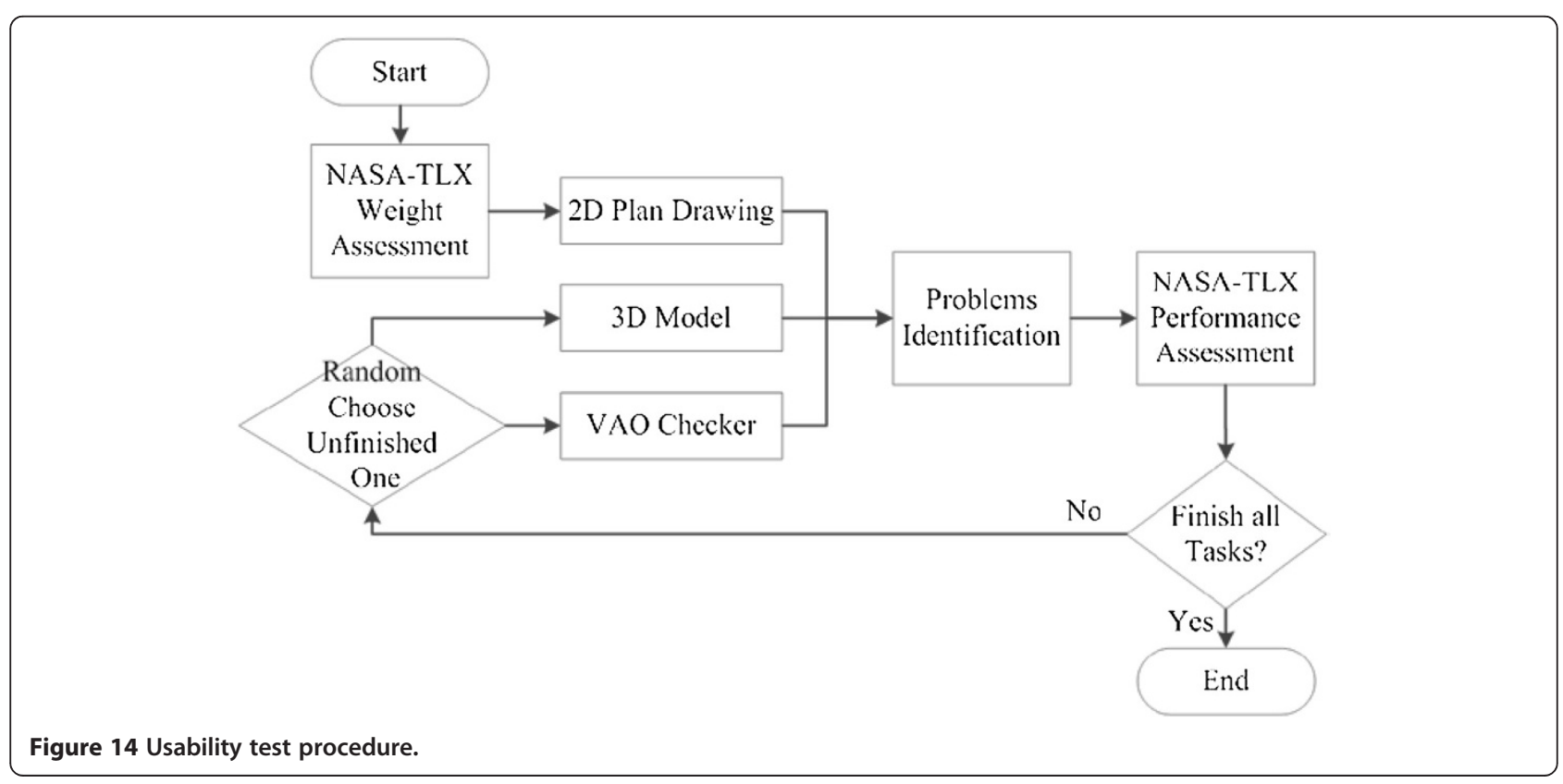




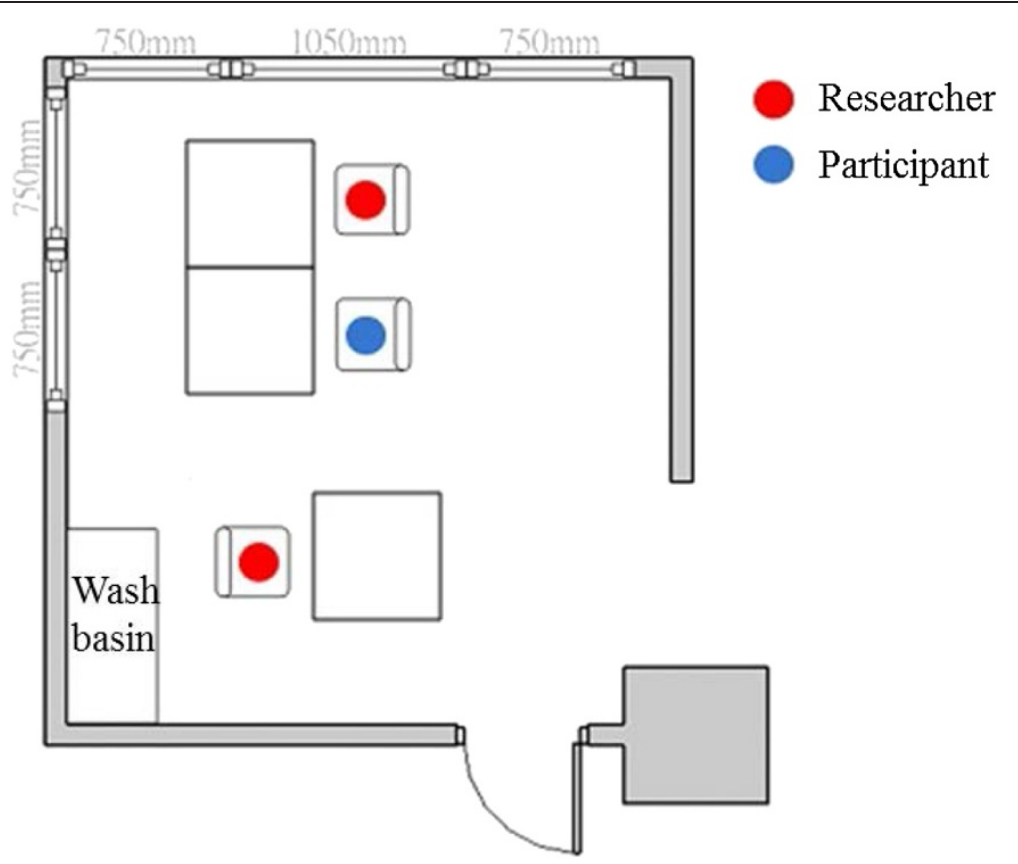

Figure 15 The test environment.

user interface and visualization information as a tool to provide users with complete information about pipeline accessibility.

\section{Future work}

Even though this research has made efforts on the pipeline accessibility, there are still some possibilities can be worked on in the future:
Number of levels divided for each accessibility category

Operational accessibility, for example, has more than one kind of controls, such as toggle switches, pushbuttons, and rotary controls. Each demands a different level of sophistication, and might need a different description for the mathematical model of operational accessibility. From the standpoint of plan amendments, VAO Checker could be much more practical tool if the

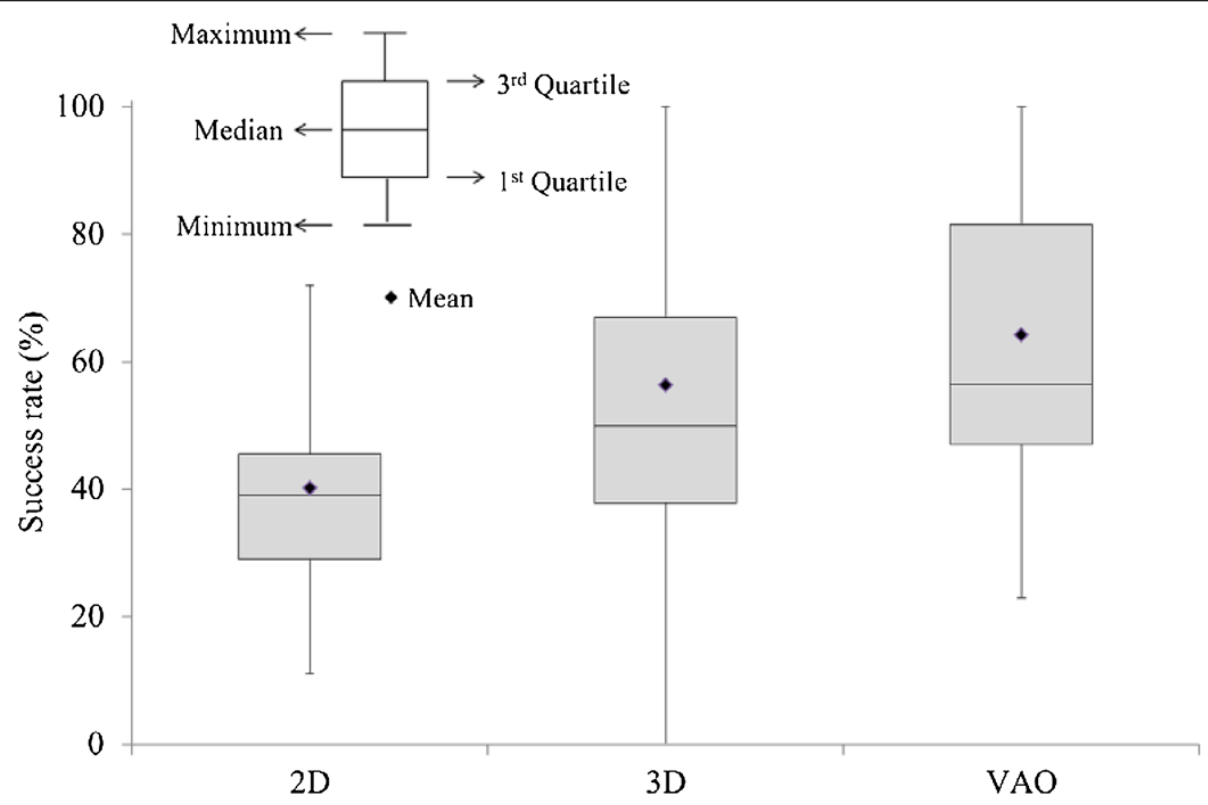

Figure 16 Success rate of each medium. 
Table 8 Statistical analysis of correctness

\begin{tabular}{lllll}
\hline Medium & Mean (\%) & Std. Deviation (\%) & p-value & \\
\hline 2D plan drawing & 40.1 & 16.3 & 2D \& 3D & $0.002^{*}$ \\
3D model & 56.4 & 25.3 & 2D \& VAO & $0.000^{*}$ \\
VAO Checker & 64.3 & 24.5 & 3D \& VAO & 0.139 \\
\hline
\end{tabular}

*the data is statistically significant.

analysis result can show the segmented and numerical degree of accessibility.

\section{Optimization via operators}

The system we propose uses computation to suggest a suitable path by considering approachable accessibility. It might become much more usable if the pipeline's designer can improve results through specific operators based on the designer's experience. Furthermore, the tremendous amount of information saved in the layout database might be referenced for future optimization.

\section{Development of pipe assembly planning}

The pipe-routing design still relies on designer's experience. We hope this system can be extended to pipe assembly planning areas for efficient implementation, which might lead to a process of automatic pipe routing. The pipeline arrangement will only become more complex in the future, and pipe assembly planning will help increase the effectiveness and efficiency of routing design.

\section{Conclusion}

This research developed a systematic method to evaluate the accessibility of pipeline maintenance. During the
Table 9 Statistical analysis of performance

\begin{tabular}{lllll}
\hline Medium & $\begin{array}{l}\text { Mean } \\
\text { (points) }\end{array}$ & $\begin{array}{l}\text { Std. Deviation } \\
\text { (points) }\end{array}$ & p-value & \\
\hline 2D plan drawing & 36.0 & 13.5 & 2D \& 3D & $0.000^{*}$ \\
3D model & 53.8 & 17.0 & 2D \& VAO & $0.004^{*}$ \\
VAO Checker & 48.0 & 17.3 & 3D \& VAO & $0.020^{*}$ \\
\hline *the data is statistically significant. & & &
\end{tabular}

early stage of this research, we interviewed six experts to determine the requirements of pipeline design. After combining the opinion of experts with a literature review, we mainly focused our research on pipeline accessibility during operation and maintenance, which is rarely discussed in previous studies. We first divided pipeline accessibility into three categories, developed mathematical models, and discussed the ergonomic details of each different category. We then developed a system called VAO Checker, which integrated the user interface and visualization information as a tool to implement the proposed methodology. VAO Checker used a simple motion-planning algorithm to find a path with acceptable approachable accessibility, and programmed the mathematical models into visualization information indicating the visual and operational accessibility. We created an example case to validate the practicality of VAO Checker, and conducted a usability test to evaluate the effectiveness of this tool. The result showed that it is a useful system for pipeline designers and engineers. It considered the pipeline accessibility within multi-pipes and enhanced the spatial comprehension. The system can be further integrated into BIM software as an API, extended to pipe assembly planning areas, or even referenced for future optimization.

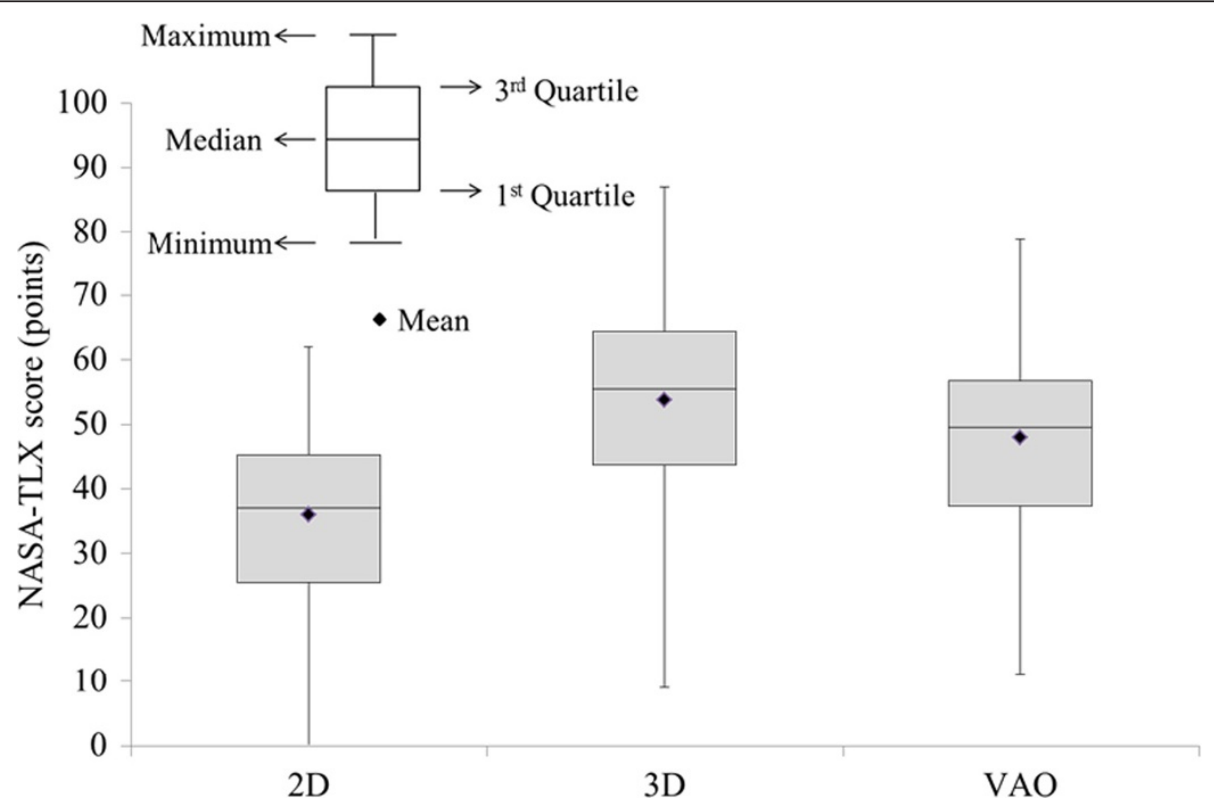

Figure 17 NASA-TLX score of each medium. 


\section{Endnotes}

${ }^{a}$ Lewis, Clarence Irving and Leibniz, Gottfried Wilhelm (1918). A survey of symbolic logic, University of California Press.

\section{Competing interests}

The authors declare that they have no competing interests.

\section{Authors' contributions}

$\mathrm{CH}$ developed the methodology and the mathematical models, programmed VAO Checker, carried out the usability test, analyzed the results and drafted the manuscript. MH assisted the literature review and the usability test. SC offered suggestion and guidance to the research. All authors read and approved the final manuscript.

\section{Acknowledgement}

This research was supported in part by Taiwan's Ministry of Economic Affairs, under contract 101-EC-17-A-15-S1-223. The authors are grateful to Mr. Ching-Yang Kao and Mr. Ming-Fa Lin of United Microelectronics Corporation (UMC), Mr. Yuan-Fu Liao and Mr. Yi-Ti Tsai of CTCl Corporation, Mr. Chien-Chih Lai of L\&K Engineering Corporation and Mr. Ching-Hsien Lee of Research Center for BIM, National Taiwan University for their kind support and assistance in this research. We like to express our appreciation to the interviewees of these corporations and organizations.

\section{Author details}

${ }^{1}$ Department of Civil Engineering, National Taiwan University, Taipei, Taiwan. ${ }^{2}$ Center for Weather Climate and Disaster Research, National Taiwan University, Taipei, Taiwan. ${ }^{3}$ Department of Civil Engineering, National Taiwan University, Taipei, Taiwan.

\section{Received: 30 December 2013 Accepted: 5 June 2014}

Published online: 27 September 2014

\section{References}

Biehl, WH, \& Inman, JA. (2010). Energy optimization for water systems. Journal of American Water Works Association, 102, 6.

Calixto, EES, Bordeira, PG, Calazans, HT, Tavares, CAC, \& Rodriguez, MTD. (2009). Plant design project automation using an automatic pipe routing routine. Computer Aided Chemical Engineering, 27, 807-812. doi: 10.1016/S1570-7946 (09)70355-4.

Chang, HS, Kang, SC, \& Chen, PH. (2009). Systematic procedure of determining an ideal color scheme on 4D models. Advanced Engineering Informatics, 23(4), 463-473. doi: 10.1016/j.aei.2009.05.002.

Chen, YH, Tsai, MH, Kang, SC, \& Liu, CW. (2013). Selection and evaluation of color scheme for 4D construction models. Journal of Information Technology in Construction, 18, 1-19.

Deliang, L, \& Huibiao, L. (2009). Interfere-check applying to 3D automatic pipe route arrangement. Proceedings of International Conference on Computational Intelligence and Software Engineering, Wuhan, 11-13. doi:10.1109/ cise.2009.5365920.

Feng, H, Fu, Y, \& Li, L. (2012). Layout space modeling for automation design of pipeline system. Proceedings of 2012 International Conference on Mechatronics and Automation (ICMA), Chengdu, 5-8. doi:10.1109/icma.2012.6283259.

Gao, Z, Walters, RC, Jaselskis, EJ, \& Wipf, TJ. (2006). Approaches to improving the quality of construction drawings from owner's perspective. Journal of Construction Engineering and Management, 132(11), 1187-1192. doi: 10.1061/ (asce)0733-9364(2006)132:11(1187).

Grootjans, R. (2009). XNA 3.0 Game Programming Recipes: A Problem-Solution Approach. New York: Apress.

Guirardello, R, \& Swaney, RE. (2005). Optimization of process plant layout with pipe routing. Computers and Chemical Engineering, 30(1), 99-114. 10.1016/j. compehemeng.2005.08.009.

Ito, T. (1999). A genetic algorithm approach to piping route path planning. Journal of Intelligent Manufacturing, 10(1), 103-114. 10.1023/a:1008924832167.

Junnila, S, Horvath, A, \& Guggemos, AA. (2006). Life-cycle assessment of office buildings in Europe and the United States. Journal of Infrastructure Systems, 12(1), 10-17. doi:10.1061/(asce)1076-0342(2006)12:1(10).

Khanzode, A, Fischer, M, \& Reed, D. (2008). Benefits and lessons learned of implementing building virtual design and construction (VDC) technologies for coordination of mechanical, electrical, and plumbing (MEP) systems on a large healthcare project. Journal of Information Technology in Construction, 13, 324-342.

Kim, D, Corne, D, \& Ross, P. (1996). Industrial plant pipe-route optimisation with genetic algorithms. Lecture Notes in Computer Science, 1141, 1012-1021.

Korde, T, Wang, Y, \& Russell, A. (2005). Visualization Of Construction Data (pp. 2-4). Toronto, Canada: Proceedings of 6th Construction Specialty Conference.

Kuo, CH, Tsai, MH, \& Kang, SC. (2011). A framework of information visualization for multi-system construction. Automation in Construction, 20(3), 247-262. 10.1016/j.autcon.2010.10.003

Miller, T, \& Johnson, D. (2010). XNA Game Studio 4.0 Programming: Developing for Windows Phone 7 and Xbox 360. Boston: Addison-Wesley Professional.

Mitsuta, T, Kobayashi, Y, Wada, Y, Kiguchi, T, \& Yoshinaga, T. (1987). A knowledge-Based Approach To Routing Problems In Industrial Plant Design. In Proceedings Of 6th International Workshop on Expert Systems \& Their Applications (pp. 28-30). Avignon, France.

Nathan, A. (2006). Windows Presentation Foundation Unleashed. Indianapolis: Sams Publishing.

Newell, RG. (1972). An Interactive Approach To Pipe Routing In Process Plants (pp. 6-10). London: Proceedings of IFIP Congress 71.

Park, JH, \& Storch, RL. (2002). Pipe-routing algorithm development: case study of a ship engine room design. Expert Systems with Applications, 23(3), 299-309. 10.1016/s0957-4174(02)00049-0.

Qian, X, Ren, T, \& Wang, CE. (2008). A survey of pipe routing design. Proceedings of 2008 Chinese Control and Decision Conference, Yantai, Shandong, doi:10.1109/ccdc 2008.4598081.

Riley, DR, Varadan, P, James, JS, \& Thomas, HR. (2005). Benefit-cost metrics for design coordination of mechanical, electrical, and plumbing systems in multistory buildings. Journal of Construction Engineering and Management, 131(8), 877-889. doi: 10.1061/(asce)0733-9364(2005)131:8(877).

Rourke, PW. (1975). Development of a Three-Dimensional Pipe Routing Algorithm. Lehigh University: PhD Dissertation.

Russell, AD, Chiu, CY, \& Korde, T. (2009). Visual representation of construction management data. Automation in Construction, 18(8), 1045-1062. 10.1016/j. autcon.2009.05.006.

Schmidt-Traub, H, Köster, M, Holtkötter, T, \& Nipper, N. (1998). Conceptual plant layout. Computers \& Chemical Engineering, 1, S499-S504. 10.1016/S0098-1354 (98)00093-3.

Songer, AD, Hays, B, \& North, C. (2004). Multidimensional visualization of project control data. Construction Innovation: Information, Process, Management, 4(3), 173-190. 10.1108/14714170410815088

The American Bureau of Shipping. (2003). Guidance Notes on the Application of Ergonomics to Marine Systems. Houston: American Bureau of Shipping.

Tsai, MH, Kang, SC, \& Hsieh, SH. (2010). A three-stage framework for introducing a 4D tool in large consulting firms. Advanced Engineering Informatics, 24(4), 476-489. 10.1016/j.aei.2010.04.002

Tsai, MH, Kang, SC, \& Hsieh, SH. (2013). Lessons learnt from customization of a BIM tool for a design-build company. Journal of the Chinese Institute of Engineers, 37(2), 189-199. 10.1080/02533839.2013.781791.

Wang, CP. (2011). An Approach for Assessing Reachability of Wheelchair Users (Master Thesis). National Taiwan University.

Wangdahl, GE, Pollock, S, \& Woodward, JB. (1974). Minimum-trajectory pipe routing. Journal of Ship Research, 18(1), 44-49.

Zhou, C, \& Yin, Y. (2010). Pipe assembly planning algorithm by imitating human imaginal thinking. Assembly Automation, 30(1), 66-74. 10.1108/ 01445151011016082.

Zhu, D, \& Latombe, JC. (1991). Mechanization of spatial reasoning for automatic pipe layout design. Artificial Intelligence for Engineering, Design, Analysis and Manufacturing, 5(1), 1-20. 10.1017/S089006040000250X.

doi:10.1186/s40327-014-0006-y

Cite this article as: Lee et al:: A visual tool for accessibility study of pipeline maintenance during design. Visualization in Engineering 2014 1:6. 